\title{
Vertical jump testing in relationship to participants' height and weight for sport integration
}

\author{
Eric Shamus $^{1 *}$, Jennifer Shamus ${ }^{2}$, Arie van Duijn ${ }^{3}$ \\ ${ }^{1}$ Department of Rehabilitation Sciences, Florida Gulf Coast University, Fort Myers, Florida, USA \\ ${ }^{2}$ Select PhysicalTherapy, Pembroke Pines, Florida, USA \\ ${ }^{3}$ Department of Rehabilitation Sciences, Florida Gulf Coast University, Fort Myers, Florida, USA
}

\begin{abstract}
The vertical jump is a functional activity for sports performance. Different devices can measure and calculate vertical jump. The Vertec utilizes a jump and reach method. The jump mats calculate jump height by measuring air time. The purpose of this study was to examine the correlation between these two types of vertical jump height measurements. Also, to examine the effect of gender, height and weight may have on these measurements. A jump mat and Vertec were utilized at the student health fair of a state University in Florida. Seventy-six college students and faculty (57 males and 19 females) participated in the vertical jump height measurements. Subjects had a mean age of $21.86( \pm 6.39)$ years, mean weight of $163.11( \pm 35.39)$ pounds, and mean height of $69.20( \pm 3.88)$ inches. When measuring vertical jump, each of these two measurement techniques provided a significant correlation when examining height, weight and sex as variables. Each variable had a significant correlation. Weight alone did not have an effect on the Pearson correlation between the devices (860). Height and weight combined reduced the correlation to 735 . When testing individuals with varying heights and weights, the correlation between the devices decreased. For test retest, the same type of vertical jump height measuring device is recommended.
\end{abstract}

Keywords: Vertical jump, Height, Weight, Jump mat, Vertec

\section{Introduction}

The vertical jump is a functional sport specific activity that is a counter movement with a dorsiflexing of the ankle and flexing the knees, hips, trunk and extending of the shoulders [1]. This creates a stretch shortening cycle followed by a quick explosion of the lower extremity musculature and upward drive of the trunk and upper extremities. It can be observed in basketball when an individual dunks a basketball or blocks a shot, in volleyball when a player goes to spike a ball or block a hit, and in football when a receiver jumps for the ball or a defensive back jumps to knock the ball away. It can also be observed in many track and field events, soccer when heading a ball, and in baseball when an outfielder is jumping high over the fence to catch a fly ball. The vertical jump is a measurement of strength and anaerobic power [2]. It is a standard test utilized when comparing athletes and is an assessment for sport performance [3]. The vertical jump is utilized for measurement, training and progression assessment. When looking at plyometric jumps, the vertical jump had the highest power output, muscle recruitment and ground reaction forces [4-6].

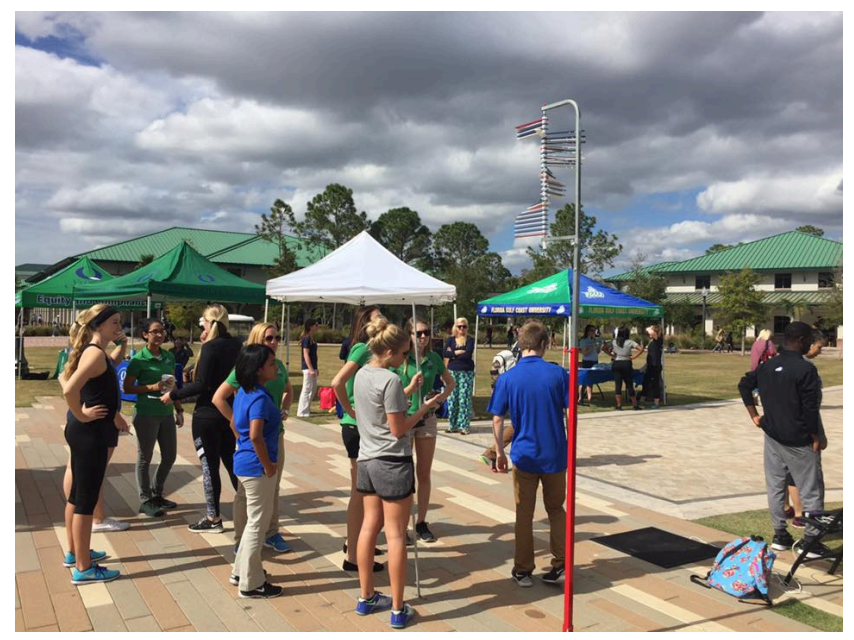

Figure 1. Vertec

A long time standard of measurement of a vertical jump has been utilizing a Vertec (see Figure 1). The Vertec is accurate to the measurement of half-inch increments. The Vertec measures the difference between standing reach and vertical jump with reach, often termed jump reach. Individuals stand flat footed and reach for the highest vane (slapstick). Then individual jumps and reaches for the highest vane (slapstick) and the difference is recorded as the vertical jump height. This is often termed the reach method. There are wall mounted and free standing portable designs. A motion analysis camera system is 
Citation: Shamus E, Shamus J, Duijn AV. Vertical jump testing in relationship to participants' height and weight for sport integration. J Phys Ther Sports Med 2018;2(1):1-4.

considered a gold standard in vertical jump height for the reach method. The video from a motion analysis system can be slowed down to observe an exact measurement of the vertical jump height. With advances in technology a variety of jump mats are on the market that measure hang time and calculate the vertical jump height. The jump mat measures to the accuracy of $1 / 10$ th of an inch. This calculation is a measurement of time off the mat, but does not take into consideration reaching ability.

Leard et al. [7] compared the jump and reach method (Vertec) and the contact mat method (Just Jump) in assessing vertical jump height when compared to a 3-camera motion analysis system. Both correlations were significant at the 0.01 level with the contact mat having closer results to the 3-camera system. The contact mat was found to be a valid measure. The Pearson $r$ between the contact mat (Just Jump) and the 3camera motion analysis system was 0.967 . The Vertec had a high correlation with the 3-camera system, but the mean differed significantly. The Pearson $r$ statistic between the jump and reach (Vertec) and the 3-camera motion analysis system and was 0.906 [7]. Buckthorpe, et al. investigated the validity of four vertical jump height measurement devices [8]. A belt mat, contact mat, portable force plate, and Vertec were compared to a criterion device, a laboratory force plate. The laboratory force plate had the highest mean value $(50.3 \mathrm{~cm})$. The portable force plate and belt mat had very close jump height values to the laboratory force plate (within $1 \mathrm{~cm}$ ). The contact mat and Vertec had significantly lower values than the laboratory force plate $(\mathrm{P}<0.001)$. The mean differences \pm were: belt mat $-0.1 \pm 5.5 \mathrm{~cm}$, contact mat $-11.7 \pm 6.4 \mathrm{~cm}$, portable force plate $-0.8 \pm 3.9 \mathrm{~cm}$, and Vertec $-2.4 \pm 6.6 \mathrm{~cm}$. The portable force plate and belt mat devices provided valid measures of vertical jump height, whereas the Vertec and contact mat devices did not in comparison to the laboratory force plate [8].

Whitmer et al. [9] simultaneously compared a vertical jump contact mat to a force plate for determining jump height and flight time. The subjects performed a vertical jump reaching for the Vertec to determine the jump height. The vertical jump mat reported greater vertical jump height than the force plate (vertical jump mat $=0.50 \pm 0.12 \mathrm{~m}$, force plate $=0.34 \pm 0.10 \mathrm{~m}$ ) and flight time (vertical jump mat $=0.629 \pm 0.078$ seconds, force plate $=0.524 \pm 0.077$ seconds). There were no significant differences of vertical jump heights from the vertical jump mat and the Vertec. Regression analyses indicated strong relationships between testing methods and suggested that high vertical jump performances may be underestimated with the vertical jump mat. The vertical jump mat may not be an appropriate tool for assessing high vertical jump performances (i.e., $\geq 0.70 \mathrm{~m} ; \approx 28$ inches) [9]. Magnúsdóttir, et al. simultaneously compared an accelerometer-based technique (KineJump), a contact mat (Newtest Powertimer 300 series), the Vertec measurement device, and a video analysis technique for measuring jump heights of subjects with a range of age (18 to 70) and athletic ability (sedentary to trained athlete) [10]. KineJump had similar results to the Vertec and contact mat, although the overall accuracy was $\pm 5 \mathrm{~cm}$. The 2 methods that rely on a time-of-flight calculation of the jump height (contact mat and accelerometer) showed lower values than the Vertec and video analysis [10].

García-López, et al. simultaneously examined the validity and reliability of a force plate (gold standard method), 2 photocell mats (SportJump System Pro and ErgoJump Plus), and a contact mat (SportJump-v1.0) with simultaneous measurements [11]. The first part showed that the 2 photocell mats underestimated the vertical jump height $(1.3 \pm 0.2 \mathrm{~cm}$ and $5.9 \pm 5.2 \mathrm{~cm}$, respectively), but only SportJump System Pro showed a high correlation with the Force Plate ( $\mathrm{r}=0.999$ and 0.676, respectively) and good intraday reliability (coefficient of variation $=2.98$ and $15.94 \%$, intraclass correlation coefficients $=0.95-0.97$ and $0.45-0.57$, respectively). There was a strong correlation $(\mathrm{r}=0.994)$ between the 2 technologies (contact vs. photocell mats) with differences in vertical jump height of $2.0 \pm 0.8 \mathrm{~cm}$ (95\% confidence interval=1.9-2.1 cm). The SportJump System Pro was a valid and reliable device. The type of mat (contact vs. photocell) affected approximately $6 \%(2 \mathrm{~cm})$ of the vertical jump height. The use of validated photocell mats instead of the contact mats was recommended [11]. Glatthorn et al. [12] examined concurrent validity and reliability of the Optojump photocell system with force plate measurements for estimating vertical jump height. Intraclass correlation coefficients (ICCs) for validity were very high (0.997 to 0.998$)$, with a systematic difference between force plate and Optojump $(-1.06 \mathrm{~cm} ; \mathrm{p}<0.001)$. Test-retest reliability of the Optojump system was excellent, with ICCs ranging from 0.982 to 0.989 , low coefficients of variation $(2.7 \%)$, and low random errors $( \pm 2.81 \mathrm{~cm})$. The Optojump photocell system demonstrated strong concurrent validity and excellent testretest reliability for the estimation of vertical jump height. Glatthorn et al. recommended the following equation that allows force plate and Optojump results to be used interchangeably: force plate jump height $(\mathrm{cm})=1.02 \times$ Optojump jump height +0.29 [12].

These studies examined the reliability and validity of the different vertical jump height measurement devices. Magnúsdóttir et al. was the only one that subdivided the subjects with a range of age (18 to 70$)$ and athletic ability (sedentary to trained athlete). When testing a general population, there are different body types in relationship to height and weight. The purpose of this study is to examine vertical jump height in relationship to individuals' height and weight and examine if there is a significant difference in vertical height measurement between a Just Jump, jump mat and a Vertec.

\section{Methods}

\section{Experimental approach to the problem}

The University has a student wide health fair in the center of campus each year. A Vertec and jump mat was utilized as a health assessment activity for the health fair. As the college students and faculty came through the health fair, they were asked if they would like to take their try at jumping. This 
approach was utilized to generalize the college population with a varying of athletic abilities, age, height and weight.

\section{Participants}

Seventy-six college students and faculty (57 males and 19 females) participated in the vertical jump height measurements. Many individuals at the health fair did not want to jump because they said they were wearing the wrong type of shoes, did not want to hurt themselves, or said they could not jump. The self-reported age of the participants ranged from 18 to 66 years old with a mean age of $21.86(+6.39)$ years. The weight of the participants ranged from 96 to 285 pounds with a mean of $163.11(+35.39)$ pounds. The height of the individuals ranged from 60 to 77 inches with a mean of $69.20(+3.88)$ inches (see Table 1). This study received Institutional Review Board approval.

Table 1. Participant Demographics.

\begin{tabular}{llll}
\hline & Mean & N & High/Low \\
\hline Age (years) & $21.86+6.39$ & 76 & $66 / 18$ \\
\hline Weight (pounds) & $163.11+35.39$ & 76 & $285 / 96$ \\
\hline Height (inches) & $69.20+3.88$ & 76 & $77 / 60$ \\
\hline
\end{tabular}

\section{Experimental procedures}

Participant height and weight was measured using a Health o Meter ${ }^{\circledR}$ Professional stadiometer and scale. If college students and faculty consented to jump, a licensed physical therapy faculty member picked a number of 1 or 2 to determine if they started with the jump mat or Vertec. Participants were given a minute to gently jump up and down to warm up. Participants jumped in sneakers or bare feet. Participants were given one try on each type of measurement. Athletic training, exercise science or physical therapy students were stationed at the jump mat and Vertec. The students were in different areas and were blinded to the results of the opposite test. A physical therapy faculty member recorded all of the results. For the jump mat, participants were instructed by an athletic training, exercise science or physical therapy student to stand in the middle of the mat and jump as high as they could and land in the center of the mat. For the Vertec, participants were instructed by an athletic training, exercise science or physical therapy student to stand flat footed and reach as high as they could with their dominant arm that would be used when jumping to reach the panes. The height was recorded and the Vertec was adjusted to allow for jump height. This was based off of asking the individual if they knew how high they could jump. If they did not, the jump height was placed in a range from 5 inches up to 15 inches above their reach. Participants were instructed to jump as high as they could and push forward the pane at the highest point.

\section{Statistical analysis}

A Pearson correlation was run between the Jump Mat and Vertec with age, height and weight taken into consideration. Correlation significance was set at the 0.01 level (2-tailed).

\section{Results}

The Vertec results demonstrated a mean jump height of 22.04 $(+5.17)$ inches. The range of the Vertec was 8.0 inches to 35.0 inches. The Jump Mat results demonstrated a mean of 22.60 $(+4.90)$ inches. The range of the Jump Mat was 10.6 inches to
34.1 inches. The Pearson correlation between the Jump Mat and Vertec was 0.868 . The correlation is significant at the 0.01 level (2-tailed).

When weight (pounds) was the controlled variable, there was a Pearson correlation between the Jump Mat and Vertec was 0.870 . The correlation is significant at the 0.01 level (2-tailed). When height (inches) was the controlled variable, there was a Pearson correlation between the Jump Mat and Vertec was 0.835 . The correlation is significant at the 0.01 level (2-tailed). When sex (male/female) was the controlled variable, there was a Pearson correlation between the Jump Mat and Vertec was 0.779 . The correlation is significant at the 0.01 level (2-tailed). When height (inches) and weight (pounds) were the controlled variable, there was a Pearson correlation between the Jump Mat and Vertec of 0.735 . The correlation is significant at the 0.01 level (2-tailed).

\section{Discussion}

The University-wide student health fair brought out a variety of the student and faculty body. Individuals ranged from nonactive individuals to club sport athletes. This helped generalize the results of the study to a more general college population.

Vertec measurements take a larger consideration into one arm reaching. This is usually functional within the sport specific activity. Two-handed standing reaching methods can be utilized, depending on the sport specific jump sequence. With single hand reach measurements to obtain the standing vertical reach, individuals one arm stretching potential is a slow reach compared to the rapid reach in the vertical jump. The single arm reach during the jump can also incorporate increased lateral trunk bending. If an individual has a shoulder reaching issue, this can limit the measurement if pain is developed when jumping and reaching. The other consideration with the Vertec is having a target. Individuals can utilize imagery and aim to assist in reaching for a higher mark. In some instances if the Vertec is not stable or tightened correctly, it can fall over and hit the individual. The Vertec measures to the accuracy of a half inch. 
Citation: Shamus E, Shamus J, Duijn AV. Vertical jump testing in relationship to participants' height and weight for sport integration. J Phys

Jump mat measurements take away the reaching component of the vertical jump. This may not be as functional of a measurement. This eliminates the over- or underestimate of the standing reach. Individuals usually don't try and reach for the sky when performing the jump test as there is not an aim point for the athlete. The jump mat measures to the $1 / 10$ th of an inch.

The overall correlation between the jump mat and the Vertec was significant $(p<0.01)$ for each of the testing variables (height, weight, sex). When weight (pounds) was the controlled variable, there was a Pearson correlation of 0.870 . The Pearson correlation decreased to 0.835 when height (inches) was the controlled variable. When sex (male/female) was the controlled variable the Pearson correlation decreased to 0.779 . When height (inches) and weight (pounds) were the controlled variable, this was the lowest Pearson correlation between the Jump Mat and Vertec at 0.735 .

Weight did not have an effect on the correlation. Height and weight combined did reduce the correlation from 0.860 to 0.735 .

\section{Practical applications}

There was a high correlation of the jump mat and Vertec results. The variables, height, weight and sex were each examined. Each variable had a significant correlation. When measuring vertical jump, each of these two measurement techniques provided a significant correlation when examining height, weight and sex as variables. Height and weight combined reduced the correlation from 0.860 to 0.735 . For test retest, the same type of vertical jump height measuring device is recommended.

\section{Statement of Authorship}

This manuscript is original and is not previously published, nor is it being considered elsewhere until a decision is made to its acceptability by the Allied Academies Journal of Physical Therapy and Sports Medicine Editorial Review Board.

\section{Funding}

This project received no funding.

\section{Conflict of Interest}

There is no conflict of interest declared. The results of the study are presented clearly, honestly, and without fabrication, falsification, or inappropriate data manipulation. The results of the present study do not constitute endorsement of the product by the authors or the Journal of Physical Therapy and Sports Medicine.

\section{Ethical Adherence}

This project does not require approval by the Institutional Review Board of Florida Gulf Coast University, as it utilizes only non-identifiable, retrospective data.

\section{References}

1. Harman EA, Rosenstein MT, Frykman PN, et al. The effects of arms and countermovement on vertical jumping. J Strength Cond Res. 1991;13(3):38-9.

2. Ostojić SM, Stojanović M, Ahmetović Z. Vertical jump as a tool in assessment of muscular power and anaerobic performance. Medicinski Pregled. 2010;63(5-6):371-5.

3. NFL Combine: Workouts and drills. Vertical Jump. Retrieved from http://www.nfl.com/combine/workouts on March 9, 2017.

4. Beneka AG, Malliou PK, Missailidou V, et al. Muscle performance following an acute bout of plyometric training combined with low or high intensity weight exercise. J Sports Sci. 2013;31(3):335-43.

5. Ebben WP, Simenz C, Jensen RL. Evaluation of plyometric intensity using electromyography. J Strength Cond Res. 2008;22(3):861-868.

6. Ebben WP, Fauth ML, Garceau LR, et al. Kinetic quantification of plyometric exercise intensity. J Strength Cond Res. 2011;25(12):3288-98.

7. Leard JS, Cirillo MA, Katsnelson E, et al. Validity of two alternative systems for measuring vertical jump height. J Strength Cond Res. 2007;21(4):1296.

8. Buckthorpe M, Morris J, Folland JP. Validity of vertical jump measurement devices. J Sport Sci. 2012;30(1):63-9.

9. Whitmer TD, Fry AC, Forsythe CM, et al. Accuracy of a vertical jump contact mat for determining jump height and flight time. J Strength Cond Res. 2015;29(4):877-81.

10. Magnúsdóttir Á, Karlsson B. Comparing three devices for jump height measurement in a heterogeneous group of subjects. J Strength Cond Res. 2014;28(10):2837-44.

11. García-López J, Morante JC, Ogueta-Alday A, et al. The type of mat (Contact vs. Photocell) affects vertical jump height estimated from flight time. J Strength Cond Res. 2013;27(4):1162-7.

12. Glatthorn JF, Gouge S, Nussbaumer S, et al. Validity and reliability of Optojump photoelectric cells for estimating vertical jump height. J Strength Cond Res. 2011;25(2): 556-60.

\section{"Correspondence to:}

Eric Shamus

Department of Rehabilitation Sciences

Marieb College of Health \& Human Services

Florida Gulf Coast University

Boulevard South

Fort Myers

Florida

Telephone (239) 590-1418

E-mail: eshamus@fgcu.edu 